\title{
STONE STABILITY UNDER DECELERATING OPEN-CHANNEL FLOW
}

\author{
Nguyen Thanh Hoan ${ }^{1}$, Rob Booij ${ }^{1}$, Bas Hofland ${ }^{2}$, Marcel J.F. Stive ${ }^{1}$ \\ and Henk Jan Verhagen ${ }^{1}$
}

The current research is aimed at finding a proper relation between flow forces acting on the bed and the bed response. To this end, experiments were carried out in which both the bed response (quantified by a dimensionless entrainment rate) and the flow field (velocity and turbulence intensity distributions) are measured. The three available stability parameters, which are used to quantify for the flow forces, were evaluated using the measured data. The focus of the evaluation is on the correlation of these stability parameters with the measured bed damage expressed in terms of the dimensionless entrainment rate. The experimental results confirm that the Shields stability parameter fails to predict bed damage for non-uniform flow conditions $\left(\mathrm{R}^{2}=0.18\right)$. In contrast, the stability parameters of Jongeling et al. (2003) and Hofland (2005) give better damage predictions $\left(\mathrm{R}^{2}=0.77\right)$. The results confirm the strong influence of the velocity and turbulence intensity distributions on the stability of bed material.

\section{INTRODUCTION}

In the design of bed protections the choice of stone sizes and weights to be used is essential. The Shields (1936) formula was developed for uniform flow conditions and is widely used to determine the required stone sizes. In the Shields formula the near-bed shear stress is the only quantity representing the flow forces on the bed. Previous studies (Jongeling et al, 2003; Hofland, 2005 and Mosselman \& Akkerman, 1998, among others) have shown that not only the near-bed shear stress (or an estimate thereof based on the mean velocity) but also the turbulence influences the stability of the bed material in flowing water. Therefore, it is important to take turbulence effects into account, especially for the design of bed protections in non-uniform flow near hydraulic structures.

\section{GOVERNING VARIABLES}

A relation between flow forces ( $\Psi$ ) acting on the bed and bed response ( $\Phi$ ) can be expressed as follows

$$
\Phi=f(\Psi)
$$

This cause-and-effect relation can be established if the flow forces and the bed response are properly described. In this section those governing variables will be discussed in details.

\footnotetext{
${ }^{1}$ Delft University of Technology, Faculty of Civil Engineering and Geosciences, Department of Hydraulic Engineering, P.O. Box 5048, 2600 GA Delft, the Netherlands, Email: H.T.Nguyen@TUDelft.NL

${ }^{2}$ WL | Delft Hydraulics, Delft, The Netherlands
} 


\section{Flow forces}

A stability parameter - expressed in the form of a dimensionless relation between hydraulic load and bed strength - is often used to quantify the influence of hydraulic forces on the bed. As the flow forces acting to move the stone (i.e., drag, shear and lift forces) are proportional to $\left(\propto \rho u^{2} d^{2}\right)$ and the force that resists movement is mainly the gravity force $\left(\equiv\left(\rho_{s}-\rho\right) g V\right)$, a general form of a stability parameter can be expressed as

$$
\Psi=\frac{u^{2}}{\Delta g d}
$$

where $\Psi$ denotes a general mobility parameter, $u$ is a typical velocity scale, $\Delta$ represents the specific submerged density of stone $\left(\equiv\left(\rho_{s}-\rho\right) / \rho, \rho_{s}\right.$ represents the density of stone, $\rho$ represents the density of water, $g$ represents the gravitational acceleration, $V\left(\propto d^{3}\right)$ represents the volume of the stone and $d$ denotes the stone diameter. For uniform flow Shields (1936) used the shear velocity $u_{*}$ to form the well-known Shields stability (mobility) parameter $\Psi_{s}$ :

$$
\Psi_{s}=\frac{u_{*}^{2}}{\Delta g d}=\frac{\tau_{b}}{\rho \Delta g d}
$$

where $\tau_{b}$ denotes the near bed shear stress $\left(\tau_{b}=\rho u_{*}^{2}\right)$. If $\Psi_{s}$ is larger than a critical value $\Psi_{c}$, movement of bed material will occur. In the Shields parameter the near-bed shear stress is the only quantity representing the flow forces on the bed. Turbulence effects are only incorporated implicitly through the empirically estimated values of $\Psi_{c}$. This is a valid approach for uniform flows, for which the ratio of turbulence intensity to $u_{*}$ is virtually constant (Hofland, 2005). In non-uniform flows, Jongeling et al. (2003) proposed a stability parameter as follow

$$
\Psi_{W L}=\frac{\left\langle(\bar{u}+\alpha \sqrt{k})^{2}\right\rangle_{h m}}{\Delta g d}
$$

where $\bar{u}$ denotes the mean velocity, $\alpha$ is an empirical parameter, $k$ represents the turbulence kinetic energy and $\langle\ldots\rangle_{h m}$ is a spatial average over a distance of $\mathrm{hm}$ above the bed. The values of $\alpha$ and $\mathrm{hm}$ were chosen so that at incipient motion of stones the stability parameter $\Psi_{W L}$ had more or less an equal value for all considered geometries. From the flow simulation it appeared that the variation of the stability parameter $\Psi_{W L}$ is smallest when a value $\alpha=6$ is selected (Jongeling et al, 2006). A value of $h m$ is chosen as

$$
h m=5 d_{n}+0.2 h
$$


where $d_{n}$ is the nominal stone diameter and $h$ is the local water depth.

Partly based on the approaches developed by Mosselman and Akkerman (1998), Hoffmans and Akkerman (1998) and Jongeling et al (2003), Hofland (2005) proposed a new method for evaluating the stability of bed protections under nonuniform flows using output of a 3D RANS model. The profiles of the mean velocity and turbulence kinetic energy in the water column above the bed are used to formulate a local stability parameter. The maximum over the depth of the local values of $(\bar{u}+\alpha \sqrt{k})$ weighted with the relative mixing length $\mathrm{Lm} / \mathrm{z}$ is used. The stability parameter, $\Psi_{L m}$, is expressed as

$$
\Psi_{L m}=\frac{\max \left[\langle\bar{u}+\alpha \sqrt{k}\rangle_{L m} \frac{L_{m}}{z}\right]^{2}}{\Delta g d}
$$

where $L_{m}$ denotes the Bakhmetev mixing length $\left(L_{m}=\kappa z \sqrt{1-z / h}\right),\langle\ldots\rangle_{L m}$ is a moving average with varying filter length $L_{\mathrm{m}}$, and $z$ is the distance from the bed. A correlation between the new mobility parameter and the bed damage was analyzed based on the data of Jongeling (2003) and De Gunst (1999). From the analysis $\alpha=6$ yielded the best collapse of data.

\section{Bed response}

Mosselman and Akkerman (1998) distinguish two ways of defining the mobility of particles: i) the number of pick-ups (n) per unit time (T) and area (A) or ii) the number of particles that is transported through a cross-secion per unit time. The former if expressed in terms of volume of entrainment is often called (volume) entrainment rate, $\mathrm{E}=\mathrm{nd}^{3} / \mathrm{AT}$. The latter is often called bed load transport, $q_{s}$. The entrainment is linked to the bed load transport by: $q_{s}=E l_{s}$ where $l_{s}$ is the displacement length. Both the entrainment rate and bed load transport can be used as bed damage indicators and are often expressed in dimensionless form as

$$
\Phi_{E}=E / \Delta g d \text { and } \Phi_{s}=q_{s} / \sqrt{\Delta g d^{3}}
$$

The use of (dimensionless) bed load transport as a bed damage indicator is conventional for uniform flow. Paintal (1971) found a strong dependence of $\Phi_{s}$ on $\Psi_{s}$ :

$$
\Phi_{s}=6.56 \times 10^{18} \Psi_{s}^{16} \quad \text { for } \quad 0.02<\Psi_{s}<0.05
$$

However, bed load transport is dependent on the hydraulics upstream; all the stones passing a certain cross section (i.e., the transport) have been entrained upstream of this section. Bed load transport is therefore considered as non-local parameter. In all cases, stability parameters are local parameters, making Eq. (1) a relation of local and non-local parameters. Such kind of relation can only be valid for uniform flow where along the channel the flow condition is unchanged. 
For non-uniform flow Hofland (2005) points out that $\Phi_{E}$ should be used as bed damage indicator because it is completely dependent on the local hydrodynamic parameters. Figure 1 shows the relations between the measured $\Phi_{E}, \Psi_{W L}$ and $\Psi_{L m}$.
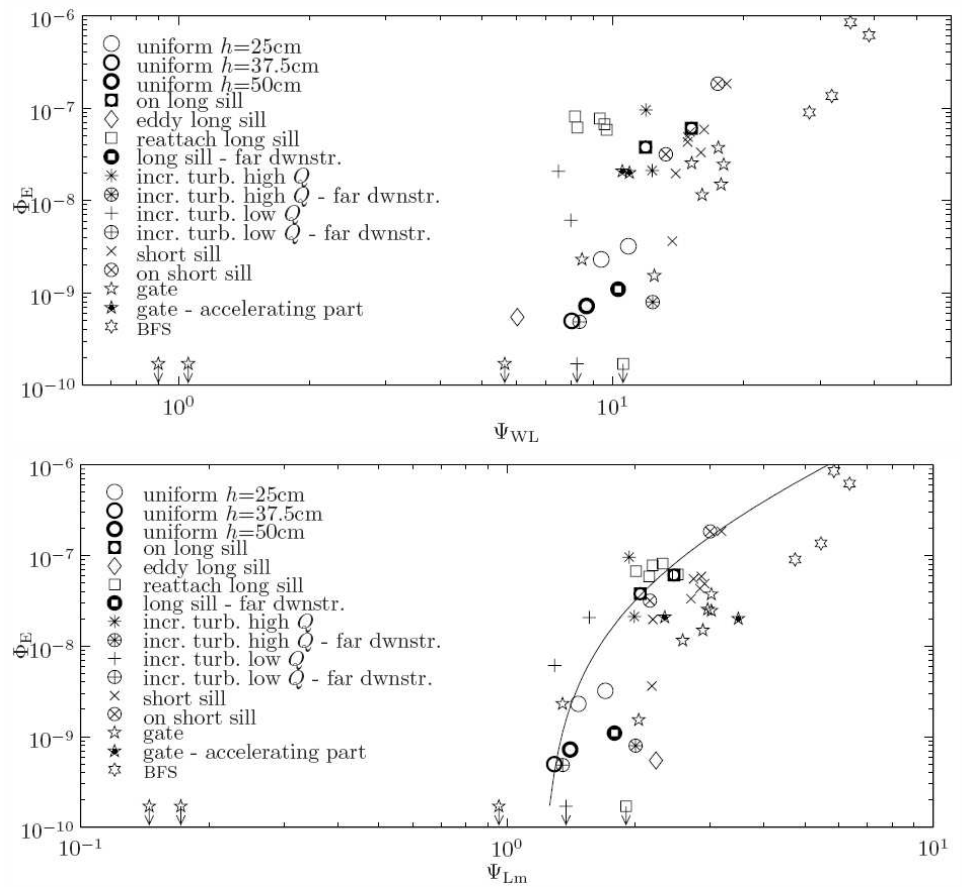

Figure 1. Top: measured $\Phi_{E}$ versus measured $\Psi_{W L}$. Bottom: measured $\Phi_{E}$ versus measured $\Psi_{L m}$ (Hofland, 2005). The curve is a tentative power law drawn more or less as an upper envelope of the data points.

\section{Concluding remarks and objectives}

From the previous review, the following conclusions can be drawn: i) for the bed damage indicator, the use of bed load transport is only suitable for uniform flow where the flow is unchanged along the channel. For non-uniform flow the dimensionless entrainment rate should be used; ii) for the stability parameters, in non-uniform flow the use of the Shields stability parameter is not sufficient as the turbulence effect is not taken into account. Two potential alternatives are the Jongeling et al parameter, $\Psi_{W L}$, and the Hofland parameter, $\Psi_{L m}$. These two stability parameters need to be verified because they were developed based on the limited data set and a large scatter is present in the $\Phi_{E}-\Psi_{W L}$ and $\Phi_{E}-\Psi_{L m}$ relations. 
This research focuses on the evaluation of the three aforementioned stability parameters and on the formulation of relation (1) for non-uniform flow based on an experimental study.

\section{EXPERIMENTS}

\section{Geometry}

The experiment was carried out in a laboratory open-channel flume with an effective length of $13.30 \mathrm{~m}$ and an available width of $0.495 \mathrm{~m}$. To decelerate the flow, an expansion is made near the end of the flume. To this end, the first part of the flume was narrowed at both sides. Then the extension was made by gradually increasing the width from the first segment to the width of the flume. By changing the expansion length (expansion angle), different combinations of velocity and turbulence can be obtained. Three different set-ups with expansion angles of 3, 5 and 7 degrees were built.

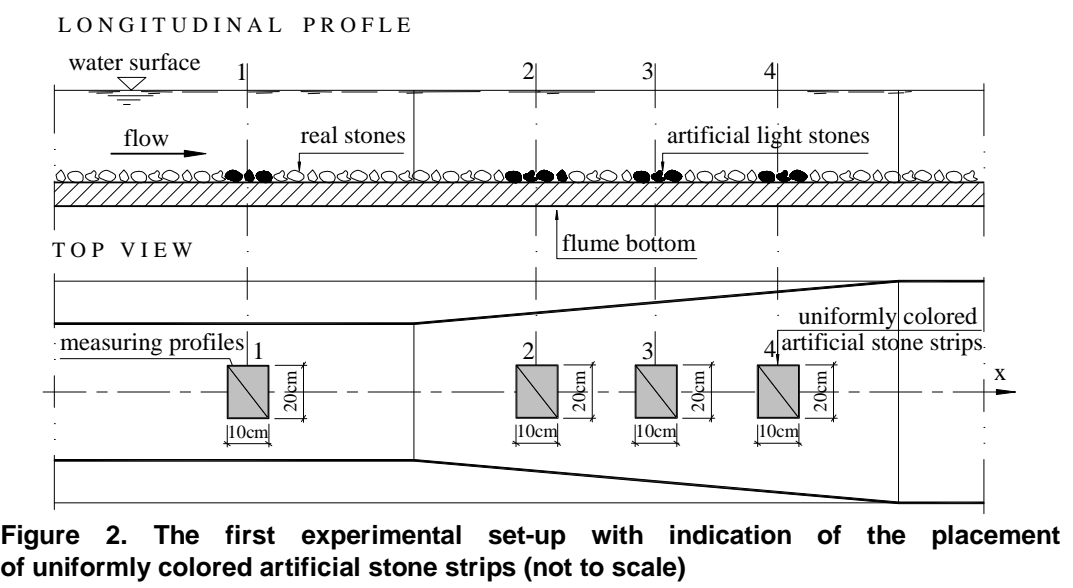

Figure 2 shows a schematic representation of the first experimental set-up. Natural stones having a nominal diameter $d_{n 50}$ of $0.80 \mathrm{~cm}$ and $d_{n 85} / d_{n 15}$ of 1.27 were used to create a 4 -cm-thick rough bottom. These stones are practically unmovable under the experimental flow conditions. To examine the stone stability, two-layer uniformly colored strips of artificial light stones were placed at the designated locations (before and along the expansion, see Figures 2 and 3 ). These stones are made of epoxy resin having densities in the range of 1320 to $2023 \mathrm{~kg} / \mathrm{m}^{3}$, mimicking shapes and sizes of natural stones. The artificial stones have a nominal diameter $d_{n 50}$ of $0.82 \mathrm{~cm}$ and $d_{n 85} / d_{n 15}$ of 1.11 .

\section{Instrumentation}

A 2-component, LDV-system was used, measuring $u$ - and $w$-components of the velocity in the streamwise vertical plane. As a light source, a Helium-Neon $(\mathrm{HeNe})$ laser with a power of $15 \mathrm{~mW}$ was used. The LDV uses the forward- 
scatter, reference-beam method. In the present study, a $400 \mathrm{~mm}$ lens was used, resulting in a measuring volume with dimensions of about $10 \mathrm{~mm}$ in spanwise direction and $1 \mathrm{~mm}$ in the other directions. Each measurement lasted 2 minutes with a sampling frequency of $500 \mathrm{~Hz}$.

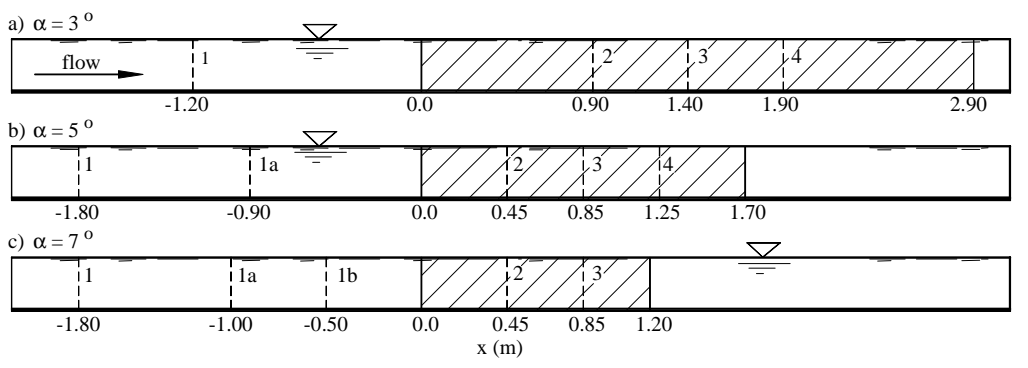

Figure 3. Longitudinal sections of the three experimental set-ups. Hatched areas depict the expansion regions. Dashed lines are stone entrainment-measurement locations.

As the flow structures in the present study are different from standard uniform open-channel flow, it is necessary to check the reliability of the LDV measurement. To this end, a measurement of a nearly-uniform open-channel flow on a smooth bed was conducted. The flow has a discharge of $12 \mathrm{l} / \mathrm{s}$, a water depth of $5.9 \mathrm{~cm}$ and $B / h=5.9$. The results are depicted in Figure 4 , showing that the data agree well with the literature.
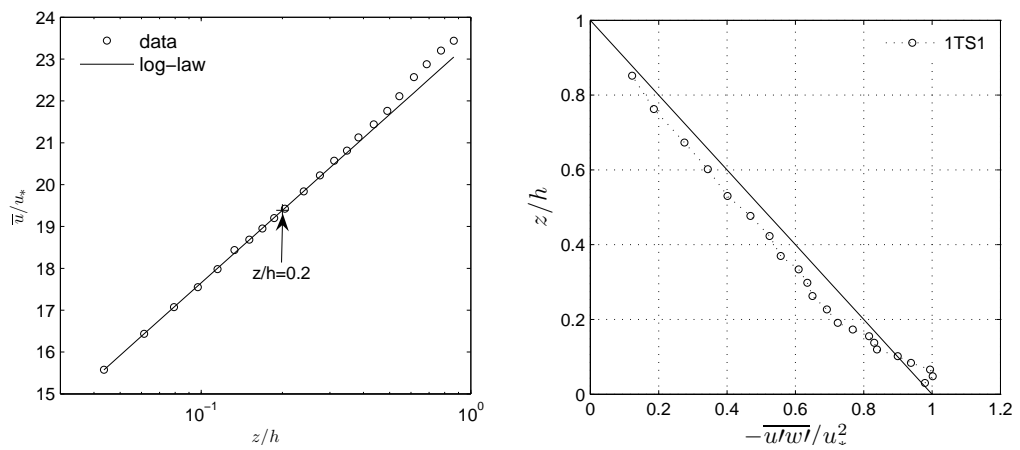

Figure 4. Distributions of velocity and Reynolds stress.

The water depth could be controlled by means of an adjustable gate at the end of the flume. The discharge could be regulated using an orifice plate in the water supply pipe. By measuring the difference in water pressure before and after the orifice plate, the discharge was determined. This pressure difference is expressed in the form of a water column height difference. Due to the pressure fluctuations, the water column height measurement has an accuracy of about 0.5 $\mathrm{cm}$. This means an accuracy of approximately $0.2 \mathrm{l} / \mathrm{s}$. The water levels were 
measured by a needle. The needle has an accuracy of $0.1 \mathrm{~mm}$. However, due to the presence of small surface waves in the flume the measurement accuracy is only about $2 \mathrm{~mm}$.

\section{Hydraulic conditions and experimental procedures}

The experiments consisted of 37 series of measurements. For the first 36 series, the artificial stones having the density from 1320 to $1384 \mathrm{~kg} / \mathrm{m}^{3}$ were used, whereas in the last series, the artificial stones with the density of $2023 \mathrm{~kg} / \mathrm{m}^{3}$ were used. The last series was dedicated to check for the influence of stone density. Of the first 36 series, twelve with different flow conditions (from A to L) were conducted for each set-up (3 set-ups). The hydraulic conditions are summarized in Table 1. The locations of the test sections are depicted in Figure 3 , namely profile 1 to profile 4 . In Table $1, Q$ is the discharge, $h$ the water depth, $\operatorname{Re}=U h / v$ the Reynolds number, $U$ mean bulk velocity, $v$ the kinematic viscosity and $F=U / \sqrt{g h}$ the Froude number. Series names consist of the setup number, the flow condition and the type of flume bottom. Series $1 \mathrm{AR}$, for example, indicates the experiment in set-up 1 with flow condition $\mathrm{A}(Q=22.0$ $1 / \mathrm{s}, \mathrm{h} h \approx 12 \mathrm{~cm}$ ) and a rough flume bottom ( $\mathrm{R}$ stands for rough, $\mathrm{S}$ stands for smooth). The twelve flow conditions are denoted as A to L (with the water depth increasing from approx. $12 \mathrm{~cm}$ to approx. $19 \mathrm{~cm}$ ). Similarly, profile names consist of the series name and a profile number, i.e., 1AR2 is the name for profile 2 of series '1AR'.

Each series consists of five repetitive measurements with the same flow conditions. The experimental procedure was as follows. First, the desired discharge was generated and the desired water depth was obtained by adjusting the weir at the downstream end of the flume. The water level and velocity measurements were carried out in the first test where the whole flume bottom was covered by natural stones. The same flow condition was reproduced and repeated for the next four tests to measure stone entrainment. To this end, the uniformly colored strips of light artificial stones were placed at the designated locations. A 30-minute initial settling period was applied prior to the actual test to remove loose stones that do not determine the strength of the bed. To start an entrainment test the flume was flooded slowly to the designated condition. After two hours, the flow was stopped and the number of displaced stones was registered. The entrainment rates obtained from the four tests are averaged to get a statistically reliable entrainment rate for the series. 
Table 1. Summary of Hydraulic conditions

\begin{tabular}{|c|c|c|c|c|c|c|c|c|c|c|c|c|c|c|}
\hline & \multirow{3}{*}{$\begin{array}{c}\alpha \\
\text { [degree] }\end{array}$} & \multirow{3}{*}{$\begin{array}{c}\mathrm{Q} \\
{[l / s]}\end{array}$} & \multicolumn{3}{|c|}{ profile 1} & \multicolumn{3}{|c|}{ profile 2} & \multicolumn{3}{|c|}{ profile 3} & \multicolumn{3}{|c|}{ profile 4} \\
\hline & & & $\mathrm{h}$ & $\mathrm{Re}$ & $\mathrm{Fr}$ & $\mathrm{h}$ & $\mathrm{Re}$ & $\mathrm{Fr}$ & $\mathrm{h}$ & $\mathrm{Re}$ & $\mathrm{Fr}$ & $\mathrm{h}$ & $\mathrm{Re}$ & $\mathrm{Fr}$ \\
\hline & & & & {$\left[10^{4}\right]$} & {$[-]$} & & {$\left[10^{4}\right]$} & {$[-]$} & & {$\left[10^{4}\right]$} & {$[-]$} & & {$\left[10^{4}\right]$} & \\
\hline 1AR & & 22 & 11.7 & 6.2 & 0.498 & 12.1 & 5.5 & 0.423 & 12.1 & 5.2 & 0.394 & 12.3 & 4.9 & 0.362 \\
\hline 1BR & & 20 & 12 & 5.7 & 0.439 & 12.1 & 5 & 0.385 & 12.2 & 4.7 & 0.357 & 12.3 & 4.4 & 0.331 \\
\hline $1 \mathrm{CR}$ & & 23 & 13 & 6.5 & 0.448 & 13 & 5.7 & 0.396 & 13.2 & 5.4 & 0.363 & 13.3 & 5.1 & 0.338 \\
\hline 1DR & & 26.5 & 13.9 & 7.5 & 0.466 & 14.3 & 6.6 & 0.395 & 14.5 & 6.2 & 0.363 & 14.5 & 5.9 & 0.343 \\
\hline 1ER & & 24 & 13.9 & 6.8 & 0.422 & 13.9 & 6 & 0.371 & 14.1 & 5.6 & 0.344 & 14.3 & 5.3 & 0.316 \\
\hline 1FR & & 27 & 15 & 7.6 & 0.425 & 14.8 & 6.7 & 0.381 & 15.2 & 6.3 & 0.346 & 15.3 & 6 & 0.323 \\
\hline $1 \mathrm{GR}$ & 3 & 31 & 15.7 & 8.8 & 0.456 & 16.1 & 7.7 & 0.385 & 16.2 & 7.3 & 0.361 & 16.2 & 6.9 & 0.338 \\
\hline $1 \mathrm{HR}$ & & 28 & 15.8 & 7.9 & 0.407 & 15.9 & 7 & 0.355 & 16.2 & 6.6 & 0.324 & 16.5 & 6.2 & 0.299 \\
\hline $1 \mathrm{IR}$ & & 31.5 & 17 & 8.9 & 0.412 & 17.1 & 7.9 & 0.359 & 17.4 & 7.4 & 0.329 & 17.8 & 7 & 0.3 \\
\hline 1JR & & 35.5 & 17.9 & 10 & 0.428 & 18.1 & 8.9 & 0.372 & 18.3 & 8.3 & 0.343 & 18.5 & 7.8 & 0.318 \\
\hline $1 \mathrm{KR}$ & & 32 & 18 & 9.1 & 0.383 & 18.1 & 8 & 0.333 & 18.3 & 7.5 & 0.308 & 18.5 & 7.1 & 0.287 \\
\hline 1LR & & 35.5 & 19 & 10 & 0.391 & 19.1 & 8.9 & 0.343 & 19.1 & 8.3 & 0.321 & 19.3 & 7.8 & 0.298 \\
\hline $2 A R$ & & 22 & 11.6 & 6.2 & 0.507 & 11.6 & 5.6 & 0.459 & 11.6 & 5.1 & 0.419 & 11.8 & 4.7 & 0.379 \\
\hline $2 \mathrm{BR}$ & & 20 & 12 & 5.7 & 0.442 & 11.9 & 5.1 & 0.401 & 11.8 & 4.7 & 0.373 & 11.8 & 4.3 & 0.341 \\
\hline $2 \mathrm{CR}$ & & 23 & 12.8 & 6.5 & 0.459 & 12.6 & 5.8 & 0.42 & 12.7 & 5.4 & 0.382 & 12.9 & 5 & 0.345 \\
\hline 2DR & & 26.5 & 13.8 & 7.5 & 0.471 & 13.8 & 6.7 & 0.424 & 13.7 & 6.2 & 0.391 & 13.9 & 5.7 & 0.355 \\
\hline 2ER & & 24 & 13.2 & 6.8 & 0.458 & 13.2 & 6.1 & 0.409 & 13.3 & 5.6 & 0.373 & 13.3 & 5.2 & 0.343 \\
\hline $2 \mathrm{FR}$ & & 27 & 14.4 & 7.6 & 0.448 & 14.1 & 6.9 & 0.418 & 14.2 & 6.3 & 0.379 & 14.3 & 5.8 & 0.345 \\
\hline $2 \mathrm{GR}$ & 5 & 31 & 16 & 8.8 & 0.443 & 15.9 & 7.9 & 0.402 & 15.9 & 7.2 & 0.368 & 16.1 & 6.7 & 0.335 \\
\hline $2 \mathrm{HR}$ & & 28 & 15.9 & 7.9 & 0.404 & 15.8 & 7.1 & 0.367 & 15.9 & 6.5 & 0.332 & 15.9 & 6 & 0.308 \\
\hline $2 \mathrm{IR}$ & & 31.5 & 16.9 & 8.9 & 0.413 & 16.7 & 8 & 0.379 & 16.6 & 7.3 & 0.35 & 16.8 & 6.8 & 0.318 \\
\hline $2 J R$ & & 35.5 & 17.5 & 10 & 0.442 & 18 & 9 & 0.382 & 17.9 & 8.3 & 0.351 & 18.1 & 7.6 & 0.32 \\
\hline $2 \mathrm{KR}$ & & 32 & 17.8 & 9.1 & 0.39 & 17.9 & 8.1 & 0.347 & 17.8 & 7.5 & 0.32 & 17.9 & 6.9 & 0.293 \\
\hline 2LR & & 35.5 & 18.6 & 10 & 0.405 & 18.2 & 9 & 0.375 & 18.5 & 8.3 & 0.335 & 18.6 & 7.6 & 0.306 \\
\hline $3 A R$ & & 22 & 12.1 & 6.2 & 0.474 & 12.6 & 5.4 & 0.393 & 12.8 & 4.8 & 0.343 & - & - & - \\
\hline 3BR & & 20 & 12 & 5.7 & 0.438 & 12.7 & 5 & 0.351 & 13 & 4.4 & 0.303 & - & - & - \\
\hline $3 C R$ & & 23 & 12.9 & 6.5 & 0.454 & 13.3 & 5.7 & 0.379 & 13.4 & 5.1 & 0.333 & - & - & - \\
\hline 3DR & & 26.5 & 13.8 & 7.5 & 0.474 & 14.4 & 6.6 & 0.387 & 14.7 & 5.8 & 0.332 & - & - & - \\
\hline 3ER & & 24 & 14.1 & 6.8 & 0.411 & 14.7 & 5.9 & 0.34 & 15 & 5.3 & 0.292 & - & - & - \\
\hline 3FR & & 27 & 14.9 & 7.6 & 0.428 & 15.5 & 6.7 & 0.355 & 15.6 & 5.9 & 0.31 & - & - & - \\
\hline $3 G R$ & 7 & 31 & 15.7 & 8.8 & 0.456 & 16.4 & 7.7 & 0.373 & 16.8 & 6.8 & 0.319 & - & - & - \\
\hline $3 \mathrm{HR}$ & & 28 & 15.8 & 7.9 & 0.406 & 16.4 & 6.9 & 0.336 & 16.6 & 6.2 & 0.294 & - & - & - \\
\hline $3 \mid \mathrm{R}$ & & 31.5 & 16.9 & 8.9 & 0.412 & 17.8 & 7.8 & 0.335 & 17.5 & 6.9 & 0.305 & - & - & - \\
\hline 3JR & & 35.5 & 17.5 & 10 & 0.442 & 18 & 8.8 & 0.37 & 18.5 & 7.8 & 0.316 & - & - & - \\
\hline $3 \mathrm{KR}$ & & 32 & 17.7 & 9.1 & 0.391 & 18 & 7.9 & 0.335 & 18.2 & 7 & 0.292 & - & - & - \\
\hline $3 \mathrm{LR}$ & & 35.5 & 18.3 & 10 & 0.414 & 19.2 & 8.8 & 0.336 & 19.5 & 7.8 & 0.293 & - & - & - \\
\hline $3 \mathrm{MR}$ & & 36 & 12.4 & 10.2 & 0.752 & 11.8 & 8.9 & 0.71 & 12.7 & 7.9 & 0.562 & - & - & - \\
\hline
\end{tabular}




\section{RESULTS}

A detailed analysis of the flow data in Hoan et al, (2007) has shown that the turbulence intensity distributions deviate from the empirical curve reported for uniform flow. The flow is non-equilibrium and the ratio of turbulence intensities to the shear velocity cannot be expressed by any universal function. Therefore, the turbulence effect on the stability of stone should be modeled explicitly. In this paper, the correlation between the available mobility parameters and the measured entrainment rate was analyzed.

Relation between $\Psi_{s}$ and $\Phi_{E}$

The shear velocity is needed to calculate the measured Shields stability parameter (Eq. (3)). This can be determined in the three following ways: i) $u_{*_{1}}$ from the Reynolds stress distribution; ii) $u_{*_{2}}$ from the log-law applied to the flow near the bottom, and iii) $u_{*_{3}}$ from the water level slope, i.e., $u_{*_{3}}=\sqrt{g h i}$. Of the three methods the last one is considered less reliable because of the uncertainty in the accuracy of the water level measurement and the unknown sidewall influence. Because of the presence of small surface waves, this third method will not be used. The values of $u_{*_{1}}$ and $u_{*_{2}}$ for all flow conditions are presented in Hoan et al (2007), showing that $u_{*_{1}}$ and $\boldsymbol{u}_{*_{2}}$ are in fairly good agreement. Correlation analysis between $\Psi_{s}$ and $\Phi_{E}$ shows less scatter when $u_{* 1}$ is used.

Table 2 summarizes the correlation analysis between the measured Shields stability parameter and the measured dimensionless entrainment rate for set-up 1, 2, 3 and all set-ups. The results show that the correlation become less when the expansion angle is larger (i.e., the flow is more non-uniform). Figure 5a shows the correlation between the Shields stability parameter and the dimensionless entrainment rate for all data. The coefficient of determination $\mathrm{R}^{2}$ $=0.18$, showing that virtually no correlation is found to exist between $\Psi_{s}$ and $\Phi_{E}$ for non-uniform flow. This clearly shows that the near bed shear stress only is not sufficient to quantify for the flow forces acting on the bed.

Table 2. Coefficient of determination

\begin{tabular}{|c|c|c|c|c|}
\hline & \multicolumn{5}{|c|}{ set-up } \\
\cline { 2 - 5 } & 1 & 2 & 3 & $1+2+3$ \\
\hline Expansion angle, $\alpha$ & 30 & 50 & 70 & \\
\hline No of data & 48 & 60 & 60 & 168 \\
\hline $\mathrm{R}^{2}$ & 0.48 & 0.21 & 0.08 & 0.18 \\
\hline
\end{tabular}



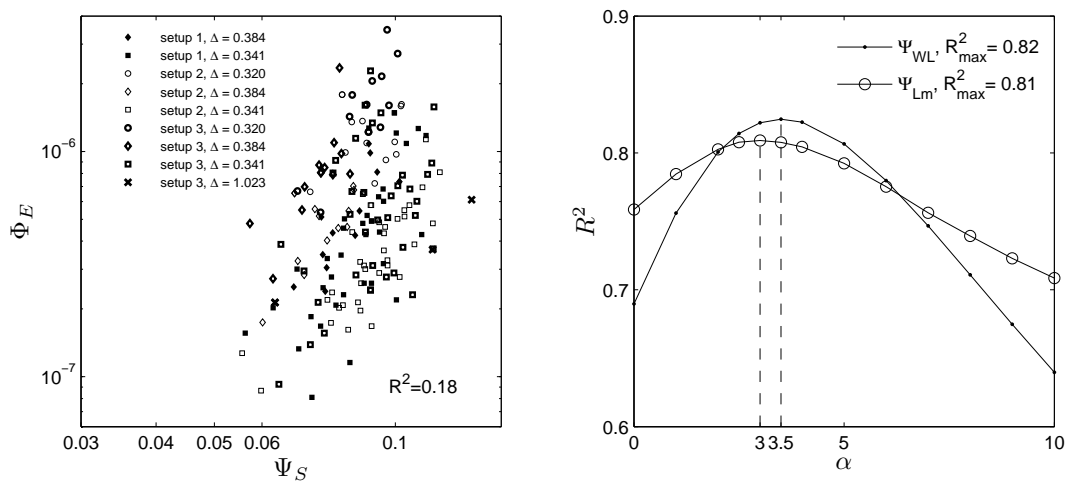

Figure 5. a) [left] measured $\Psi_{s}$ versus measured $\Phi_{E}$. b) [right] Sensitivity analysis

of $\alpha$ in Eqs. (4) and (6).

Relation between $\Psi_{W L}, \Psi_{L m}$ and $\Phi_{E}$

As only two velocity components ( $u$ - streamwise and $w$ - upward) are available, the turbulence kinetic energy in Eqs (4) and (6) was approximated by assuming that $\sigma(v)=\sigma(u) / 1.9$. The approximation is based on the EMS measurement of the flow conditions where both $u$ - and $v$-velocity components were measured. The results show that, in contrast to the Shields stability parameter, the Jongeling et al. and Hofland stability parameters are strongly correlated to the measured dimensionless entrainment parameter:

$$
\begin{gathered}
\Phi_{E}=5.4 \times 10^{-14} \Psi_{W L}^{4.9} \quad \text { for } \quad 18<\Psi_{W L}<40\left(R^{2}=0.78, \alpha=6\right) \\
\Phi_{E}=1.2 \times 10^{-9} \Psi_{L m}^{4.5} \quad \text { for } \quad 2.5<\Psi_{L m}<5.5\left(R^{2}=0.77, \alpha=6\right)
\end{gathered}
$$

In Eqs (9) and (10) the turbulence magnification factor $\alpha$ of 6 was used as suggested by Jongeling et al (2003). This was chosen based on the smallest variation of the stability parameter $\Psi_{W L}$ at the incipient motion state. In this paper we will use another criterion to choose the value of $\alpha$. The value of $\alpha$ that give the best correlation between the stability parameters and the dimensionless entrainment rate will be chosen. This sensitivity analysis of $\alpha$ in $\Psi_{W L}$ and $\Psi_{L m}$ is shown in Figure 5b. It turns out that $\alpha=3.5$ (for $\Psi_{W L}$ ) and $\alpha=3.0$ (for $\Psi_{L m}$ ) give the best correlation $\left(R^{2} \approx 0.81\right.$ for both). Figure 6 shows the correlation between these two stability parameters and the dimensionless entrainment rate. The results also confirm that the influence of stone density is well incorporated in the formula. The relations between the two modified stability parameters and the entrainment rate are expressed as follow:

$$
\Phi_{E}=1.16 \times 10^{-12} \Psi_{W L}^{4.57} \quad \text { for } 10<\Psi_{W L}<25\left(R^{2}=0.82, \alpha=3.5\right)
$$



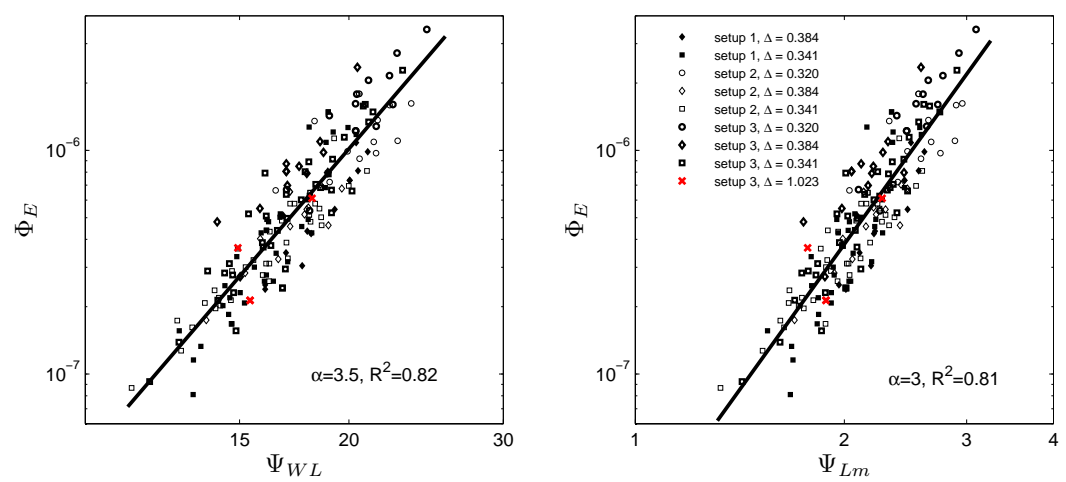

Figure 6. Left: measured $\Psi_{W L}$ versus measured $\Phi_{E}$. Right: measured $\Psi_{L m}$ versus measured $\Phi_{E}$

$$
\Phi_{E}=1.9 \times 10^{-8} \Psi_{L m}^{4.32} \quad \text { for } \quad 1.3<\Psi_{L m}<3.2\left(R^{2}=0.81, \alpha=3\right)
$$

\section{CONCLUSIONS}

1. The experiments reported herein indicate that the Shields stability parameter is not sufficient for presenting the flow forces acting on the bed in nonuniform flow.

2. Jongeling et al $\left(\Psi_{W L}\right)$ and Hofland $\left(\Psi_{L m}\right)$ stability parameters properly represent the flow forces on the bed and these parameters can be successfully used to predict bed damage, i.e., using Eqs (11) and (12). $\alpha=$ 3.5 (for $\Psi_{W L}$ ) and $\alpha=3.0$ (for $\Psi_{L m}$ ) give the best correlation to the measured entrainment rate.

3. Based on these experiments, no preference for $\Psi_{W L}$ or $\Psi_{L m}$ can be found.

4. The results confirm the strong influence of the velocity and turbulence intensity distributions on the stability of bed material.

\section{REFERENCES}

De Gunst, M. (1999). Stone stability in a turbulent flow behind a step. M.Sc. thesis, Delft University of Technology, Delft.

Hoan, N. T., Booij, R., Stive, M. J., Verhagen, H. J. (2007). Decelerating openchannel flow in a gradual expansion. In Asian and Pacific Coasts 2007. Nanjing, China.

Hoffmans, G. J. C. M., \& Akkerman, G. (1998). Influence of turbulence on stone stability. In 7th international conference on River Sedimentation. 
Hofland, B. (2005). Rock \& roll: turbulence-induced damage to granular bed protections. Ph.D. thesis, Delft University of Technology.

Jongeling, T.H.G, Blom, A., Jagers, H.R.A, Stolker, C., Verheij, H.J. (2003). Design method granular protections (Technical Report Q2933 / Q3018). WL| Delft Hydraulics. (In Dutch)

Jongeling, T.H.G., Jagers, H.R.A., Stolker, C., \& Verheij, H.J. (2006). Design of granular bed protections using a RANS 3D-flow model. The Third International Conference on Scour and Erosion, Amsterdam, The Netherlands.

Mosselman, E., Akkerman, G. J. (1998, October). Low-mobility transport of course grained material. (Tech. Rep. No. Q2395.40). WL|Delft Hydraulics.

Paintal, A. S. (1971). Concept of critical shear stress in loose boundary open channels. Journal of Hydraulic Research, 9(3), 91-113.

Shields, A. (1936). Anwendung der Aehnlichkeitsmechanik und der Turbulenzforschung auf die Geschiebebewegung. Mitteilungen der Preussischen Versuchsanstalt fur Wasserbau und Schiffbau, Heft 26, Berlin. (in German) 
KEYWORDS - CSt07

Abstract acceptance number: 204

STONE STABILITY UNDER DECELERATING OPENCHANNEL FLOW

$1^{\text {st }}$ Author: Nguyen Thanh Hoan

$2^{\text {nd }}$ Author: Rob Booij

$3^{\text {rd }}$ Author: Bas Hofland

$4^{\text {nd }}$ Author: Marcel J.F. Stive

$5^{\text {nd }}$ Author: Henk Jan Verhagen

Stone stability

Bed protection

Bed damage

Stone entrainment

Bed load transport

Turbulence

Decelerating flow

Open-channel flow 